\title{
A FEASIBILITY STUDY ON INCREMENTAL BUNDLE ADJUSTMENT WITH FISHEYE IMAGES AND LOW-COST SENSORS
}

\author{
Antonio M. G. Tommaselli ${ }^{1}$, Mariana B. Campos $^{1}$, Letícia F. Castanheiro ${ }^{1}$ and Eija Honkavaara ${ }^{2}$ \\ ${ }^{1}$ Department of Cartography, School of Technology and Sciences, São Paulo State University (UNESP), \\ São Paulo 19060-900, Brazil \\ ${ }^{2}$ Department of Remote Sensing and Photogrammetry of the Finnish Geospatial Research Institute FGI, Geodeetinrinne 2, FI-02430 \\ Masala, Finland
}

\section{Commission II}

KEY WORDS: Low-cost Navigation Sensors, MEMS, Poly-dioptric System, Personal Mobile Terrestrial System

\begin{abstract}
:
Low cost imaging and positioning sensors are opening new frontiers for applications in near real-time Photogrammetry. Omnidirectional cameras acquiring images with $360^{\circ}$ coverage, when combined with information coming from GNSS (Global Navigation Satellite Systems) and IMU (Inertial Measurement Unit), can efficiently estimate orientation and object space structure. However, several challenges remain in the use of low-cost sensors and image observations acquired by sensors with non-perspective inner geometry. The accuracy of the measurement using low-cost sensors is affected by different sources of errors and sensor stability. Microelectromechanical systems (MEMS) present a large gap between predicted and actual accuracy. This work presents a study on the performance of an integrated sensor orientation approach to estimate sensor orientation and 3D sparse point cloud, using an incremental bundle adjustment strategy and data coming from a low-cost portable mobile terrestrial system composed by off-theshelf navigation systems and a poly-dioptric system (Ricoh Theta S). Experiments were performed in an outdoor area (sidewalk), achieving a trajectory positional accuracy of $0.33 \mathrm{~m}$ and a meter level 3D reconstruction.
\end{abstract}

\section{INTRODUCTION}

The advancements in the photogrammetric data processing and the increasing number of low-cost and lightweight navigation (MEMS) and imaging sensors, available in the market have encouraged the development of new integrated sensor orientation (ISO) approaches. These sensor technologies have opened many possibilities for real-time Photogrammetry considering several sets of observations from different sources. Real-time and mobile Photogrammetry requires an incremental approach for positioning and reconstruction with bundle adjustment (BA), since new measurements are made available at each time step during sensor traversing. An incremental BA can be understood as an estimation technique based on a sequential adjustment for determination of image orientation and 3D points' coordinates, in order to obtain an optimal least square solution (Gruen, 1988). A reasonable computational cost for a real-time solution requires a dynamic data acquisition and suitable initial information of the sensor pose and tie point coordinates. Initial information provided by GNSS/IMU systems can improve the processes having high computational requirements, such as image matching and bundle adjustment.

Low cost commercial cameras with fisheye lenses (e.g. Go Pro $360^{\circ}$, Ricoh Theta, Insta360, Samsung Gear360, Nikon Keymission 360 and LG360.) and catadioptric systems (e.g. VSN Mobil V. $360^{\circ}$ ) have motivated the use of large field of view images in close range Photogrammetry (CRP), especially for indoor robot navigation (Lourenco et al., 2012) and outdoor mapping applications, such as urban mapping and ground vehicles automation (Scaramuzza et al., 2009) (MurArtal et al., 2015) (Suhr et al., 2017), forest monitoring (Campos et al., 2018) and precision agriculture (Ericson and Åstrand, 2010; Cheein et al., 2011). Omnidirectional systems are an attractive alternative due to large field of view around the sensor, which allows more features to be tracked in a single image shot. However, using these new navigation and imaging sensors requires adaptations in the photogrammetric processes to support applications in CRP. Omnidirectional images have huge scale and illumination variations between scenes and large radial distortion, which directly affects photogrammetric processes, such as image matching and bundle adjustment. Furthermore, measurements generated by low cost MEMS sensors present large noise and there is a substantial gap between their predicted and actual performances.

Therefore, this paper presents an ISO approach to estimate sensor orientation and a sparse 3D point cloud from data acquired by a low-cost portable mobile terrestrial system (PMTS), using an incremental bundle adjustment strategy consistent with the fisheye lens geometry.

\section{PERSONAL MOBILE MAPPING SYSTEM SETUP}

The mobile terrestrial system used in this research is a PMTS, which can be defined as a set of sensors embedded in a backpack platform that are carried by a human operator (Figure 1.a). PMTS approaches have been increasingly used in CRP applications, due to the dynamic data acquisition on ground perspective, in places with difficult access, such as forest, agricultural fields, sidewalks and indoor buildings. The proposed PMTS consists of miniaturized and off-the-shelf navigation sensors and an omnidirectional camera (Figure 1.b).

The navigation system, which is composed of a GPS receiver (Ublox NEO-6M) and an IMU (MPU 6050) integrated with an Arduino microprocessor. Ublox NEO-6M is a low-cost single frequency GPS (global positioning system) receiver that provides real-time position (latitude, longitude and geometric height), velocity and time data in the NMEA (National Marine Electronics Association) protocol with a meter level planialtimetric accuracy, while, MPU 6050 measures angular velocities and accelerations for $\mathrm{X}, \mathrm{Y}$ and $\mathrm{Z}$ axis. Therefore, yaw, pitch and roll angles can be estimated. 
The Ricoh Theta $\mathrm{S}$ is a polydioptric system composed of two cameras with fisheye lenses (sensor 1 and sensor 2) specially arranged in the same structure in a back-to-back position to cover a full spherical field of view, aiming at $360^{\circ}$ image generation (Figure 1.b). Ricoh Theta $\mathrm{S}$ acquires 29 dual-fisheye frames per second in video mode with a fisheye image composed of $960 \times 1080$ pixels for each sensor. Therefore, the PMTS affords two fisheye images (one for each sensor) and initial exterior orientation parameters (EOPs) for each one second of data collection. More details about the PMTS setup can be found in Campos et al. (2018).

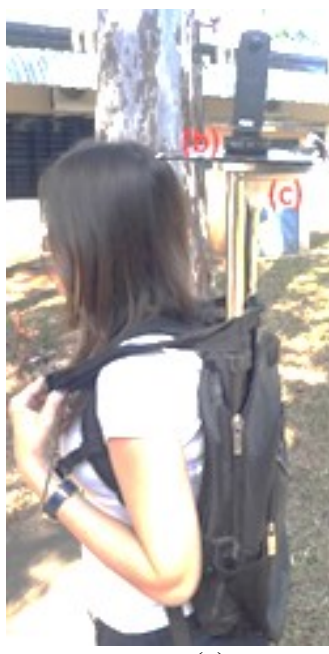

(a)

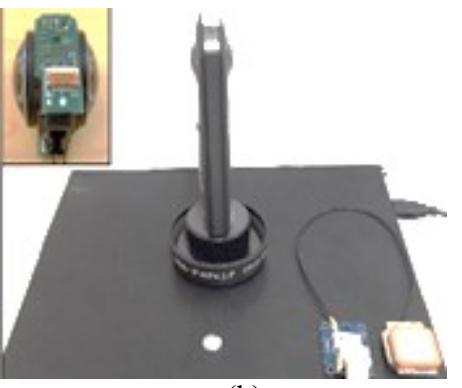

(b)
Figure 1. Personal mobile terrestrial system (PMTS) for a sequential data acquisition: (a) PMTS carried by an operator, (b) Ricoh Theta S camera (Campos et al., 2019).

\section{MATHEMATICAL MODEL}

The image acquisition using polydioptric systems based on dual-fisheye lenses does not follow the collinearity condition, requiring a suitable functional model to reflect the behaviour of the fisheye lenses (Hughes et al., 2010). Furthermore, the relative orientation between cameras needs to be considered for a rigorous approach. The mathematical model used in the incremental bundle adjustment is based on the equidistant projection (Equation 1). Concerning the offsets and misalignment angles between the two fisheye lenses of the cameras composing Ricoh Theta S, constraints based on stability of the relative orientation parameters (ROPs) between cameras (base elements $\mathrm{Bx}, \mathrm{By}, \mathrm{Bz}$ and rotation matrix $\mathrm{R}^{\mathrm{R}}$ ) were added in the bundle adjustment for a simultaneous estimation of the EOPs from sensor 1 and sensor 2 (Tommaselli et al., 2013). The simultaneous bundle adjustment with relative orientation constraints is based on the condition that ROP are stable during the same image acquisition process, but small variations are admitted as random variables (Equations 2 and 3) (Tommaselli et al., 2013). This approach was implemented in $\mathrm{C} / \mathrm{C}++$ language on the $\mathrm{CMC}$ (Calibration of Multiple Cameras) program, based on (Tommaselli et al., 2013) and (Marcato Junior et al., 2015). Equation 1 represents the equidistant mathematical model, in which the image coordinates $\left(\mathrm{x}_{\mathrm{f}}, \mathrm{y}_{\mathrm{f}}\right)$ are related to the principal distance (c) and Xc, Yc and Zc (the 3D point coordinates in the photogrammetric reference system) (Lowe, 2004). The relationship of these 3D points coordinates with the ground coordinates system requires a similarity transformation as a function of the perspective centre coordinates and Euler angles (Marcato Junior et al., 2015), which will be embedded in Equation 1.

$$
\begin{gathered}
\mathrm{x}_{\mathrm{f}}=-\mathrm{c} \cdot \frac{\mathrm{X}_{\mathrm{c}}}{\sqrt[2]{\mathrm{X}_{\mathrm{c}}{ }^{2}+\mathrm{Y}_{\mathrm{c}}{ }^{2}}} \cdot \arctan \left(\frac{\sqrt[2]{\mathrm{X}_{\mathrm{c}}{ }^{2}+\mathrm{Y}_{\mathrm{c}}^{2}}}{\mathrm{Z}_{\mathrm{c}}}\right) \\
\mathrm{y}_{\mathrm{f}}=-\mathrm{c} \cdot \frac{\mathrm{Y}_{\mathrm{c}}}{\sqrt[2]{\mathrm{X}_{\mathrm{c}}{ }^{2}+\mathrm{Y}_{\mathrm{c}}{ }^{2}}} \cdot \arctan \left(\frac{\sqrt[2]{\mathrm{X}_{\mathrm{c}}{ }^{2}+\mathrm{Y}_{\mathrm{c}}^{2}}}{\mathrm{Z}_{\mathrm{c}}}\right) \\
\left.\left(\left[\begin{array}{lll}
B_{X} & B_{Y} & B_{Z}
\end{array}\right]^{T}\right)^{i}-\left(\begin{array}{lll}
B_{X} & B_{Y} & B_{Z}
\end{array}\right]^{T}\right)^{i+1}=0+v
\end{gathered}
$$

\section{INCREMENTAL BUNDLE ADJUSTMENT METHODOLOGY}

The structure of the proposed incremental bundle adjustment relies on five main sequential steps (1) Data acquisition of two fisheye images (sensor 1 -S1 and sensor 2 - S2) and initial EOPs with a frequency of $1 \mathrm{~Hz}$; (2) an image matching process for fisheye images based on SIFT (Scale-Invariant Feature Transform) operator (Lowe, 2004), known as SIFT $_{\text {RFS }}$ (Campos et al., 2019); (3) The estimation of initial 3D coordinates (X, Y, $\mathrm{Z})$ of the $k$ image observation from $\mathrm{SIFT}_{\mathrm{RFS}}$, using a space intersection method adapted for fisheye lens geometry; (4) A local bundle adjustment with incremental observations, and finally; (5) the initial values of EOPs and 3D coordinates (X, Y, $\mathrm{Z}$ ) are updated with the estimated values from the bundle adjustment and respective standard deviations obtained from the covariance matrix $\left(\sigma_{\mathrm{XYZ}}, \sigma_{\mathrm{OBS}}\right)$. The image matching process and the initial map estimation were developed in MATLAB scripts, while the incremental bundle adjustment was performed with the in-the-house software $\mathrm{CMC}$, implemented in $\mathrm{C} / \mathrm{C}++$ language.

\subsection{Input data and PMTS sequential data acquisition}

The proposed methodology considers that IOPs (interior orientation parameters), ROPs and system boresight angles are known from a previous calibration process. The location of a set ground control points (GCP), especially in the begging of the trajectory, or an accurate position of the starting exposure station is recommended to enable a suitable initial solution. This external information is an input data for the incremental process. The sequential data acquisition is performed using the PMTS described in Section 2. This system enables the acquisition of omnidirectional images (dual-fisheye) and initial EOPs with $1 \mathrm{~Hz}$ frequency, ranging in a period of time $(\mathrm{t})$ between 0 (start) and $\mathrm{n}$ seconds (end). Therefore, a set of $M$ fisheye images and $M$ initial EOPs are obtained in function of the time $(M=2 * \mathrm{t})$.

\subsection{Image Matching}

The image matching process starts with $\mathrm{t}=3$ (and, thus $M=6$ ), considering an initial pair of triplets of omnidirectional images (6 fisheye images, forming 3 pairs with $360^{\circ}$ viewing angle) and 6 initial EOPs (S1 and S2). The ROPs, between S1 and S2, estimated in a previous calibration step, are $179.98^{\circ}, 0.11^{\circ}$ and $179.66^{\circ}(\Delta \omega, \Delta \varphi, \Delta \kappa$, respectively), and $0.019 \mathrm{~m}$ between cameras perspective centers. A strategy for reduction of the search space, considering the epipolar geometry on the sphere domain, was combined with SIFT to avoid outliers. This 
approach was named as SIFT $_{\text {RFS }}$ (Campos et al., 2019). For each key point extracted in the left image a search window is defined in the right image, based on the epipolar line projection. Then, the invariant descriptor vector from the key point searched is compared only with the key points belonging to that search window. The estimation of the epipolar line uses the ROPs values computed from the initial EOPs (for images taken at different exposure stations). Therefore, the search window dimensions depend on the errors in the initial EOPs. Subsequently, EOPs are recursively estimated thus improving the search window endpoints and reducing its dimensions. The use of ROPs parameters from EOPs measured with the GPS/IMU, brings many benefits for an optimized process, for instance, there is no need for system calibration, systematic errors are the same in both images, the adjustment implementation is simplified, and the relative accuracy is higher than absolute, especially using low-cost sensors. This iterative process was performed from each possible pair of images (keyframes) during image acquisition (Campos et al., 2019).

SIFT $_{\mathrm{RFS}}$ was applied in the two sets of fisheyes images from sensor 1 and sensor 2 independently. At the end of each iteration, $k$ match points coordinates in the image space are obtained, which are used as tie points in the incremental bundle adjustment. Initial 3D coordinates of the tie points in the object space (ground coordinates) are required in the bundle adjustment, to reduce iterations and the computational time.

\subsection{Initial map (approximated values for the ground coordinates of the tie points)}

Initial values for the ground coordinates of the tie points detected by SIFT $_{\text {RFS }}$ were obtained with a space intersection model adapted with a mathematical model for fisheye geometry and using the data provided by the PMTS navigation system as initial EOPs. In this case, the image coordinates are projected to an intermediary spherical surface, with $\mathrm{z}$ component being different from the principal distance. Therefore, the $z$ component of a point over a sphere is computed (Equation 4) for each observation in the photogrammetric system $(\mathrm{x}, \mathrm{y})$ as a function of the radius $(r)$ and the incident angle $(\alpha)$, which will be used to calculate the projecting vectors $u, v, w$, for the right (i) and left images (j). Then, the approximate values for the scales $\left(\lambda_{\mathrm{i}}, \lambda_{\mathrm{j}}\right)$ for each match point can be estimated solving a system of three linear equations with two unknowns (Equation 6 to Equation 8). Equation 6 is used to estimate the scales $\left(\lambda_{i, j}\right)$, which are obtained from Equation 5, according to the base elements $(B x, B y, B z)$. Equation 7 presents the vector of observation $\left(L_{b}\right)$ composed by the base elements $(B x, B y, B z)$ computed from the initial EOPs (acquired by Ublox NEO 6M), the coefficients matrix $(A)$ with the projecting vectors and the parameter vector $\left(\mathrm{X}_{\mathrm{a}}\right)$. Equation 8 presents the solution from the least squares method, estimating values for the scale factors $\left(X_{a}\right)$, in which the observations have the same weight.

$$
\begin{gathered}
r=\sqrt{\left(x^{2}+y^{2}\right)} ; \quad \alpha=\frac{r}{c} ; \quad z=r * \tan \left(\frac{\pi}{2-\alpha}\right) \\
X_{\text {proj }}^{i, j}=X_{0}^{i, j}+\lambda^{i, j} \cdot u^{i, j} ; Y_{p r o j}^{i, j}=Y_{0}^{i, j}+\lambda^{i, j} \cdot v^{i, j} ; Z_{p r o j}^{i, j}=Z_{0}^{i, j}+\lambda^{i, j} \cdot w^{i, j}(5) \\
B_{X}=\lambda^{i} \cdot u^{i}-\lambda^{j} \cdot u^{j} ; B_{Y}=\lambda^{i} \cdot v^{i}-\lambda^{j} \cdot v^{j} ; B_{Z}=\lambda^{i} \cdot w^{i}-\lambda^{j} \cdot w^{j} \quad(6) \\
L b=\left[\begin{array}{l}
B x \\
B y \\
B z
\end{array}\right] ; \quad A=\left[\begin{array}{ll}
u^{i} & -u^{j} \\
v^{i} & -v^{j} \\
w^{i} & -w^{j}
\end{array}\right] ; \quad X a=\left[\begin{array}{l}
\lambda^{i} \\
\lambda^{j}
\end{array}\right] \\
X a=\left(A^{T} \cdot P \cdot A\right)^{-1}\left(A^{T} . P . L b\right)
\end{gathered}
$$

If the scales are known $(X a)$, the $\mathrm{x}$ and $\mathrm{y}$ coordinates in the object space from the right (i) and left images (j) can be projected to the object space $\left(X_{p r o j}, Y_{p r o j}, Z_{p r o j}\right)$ by Equations 5 . The average values of $X_{\text {proj }}^{i}, Y_{p r o j}^{i}, Z_{\text {proj }}^{i}$ and $X_{\text {proj }}^{j}, Y_{\text {proj }}^{j}, Z_{\text {proj }}^{j}$, computed by space intersection, are the $3 \mathrm{D}$ coordinates of the match point in the object space $(\mathrm{X}, \mathrm{Y}, \mathrm{Z})$. This initial coordinates in the object space are computed for each set of new image observations $(k)$ from the sequential image matching process with SIFT $_{\mathrm{RFS}}$.

\subsection{Incremental bundle adjustment estimation}

The incremental approach starts with image points from the omnidirectional image triplet obtained with SIFT $_{\text {RFS. These }}$ observations, the GCPs coordinates, approximated values of 3D ground coordinates $(\mathrm{X}, \mathrm{Y}, \mathrm{Z})$, initial EOPs and the calibrated IOPs and ROPs (considered as constants), are inputs for the first iteration of the incremental bundle adjustment. Observations and parameters are combined in an adjustment performed with the unified approach of least squares method and parameters constraints (Mikhail and Ackerman, 1976), which enables the simultaneous estimation of EOPs and 3D ground coordinates for this omnidirectional image triplet. The initial values of EOPs and 3D coordinates are updated with the estimated values from the bundle adjustment and their respective standard deviations obtained from the covariance matrix. Then, an omnidirectional image pair (two fisheye images from each sensor) is added to the process with the respectively initial EOP and GCP in the next iteration. SIFT $T_{\mathrm{RFS}}$ is applied for the new keyframes. The initial map and the set of EOPs are now composed by previously adjusted and initial values. Therefore, in the incremental bundle adjustment process, parameters are organized in two sets with different weights: one with parameters that had been already estimated in the bundle adjustment in an earlier instance and a second one with new parameters observed in the scene in the current instance with initial values. The bundle adjustment process is performed again, and this incremental process repeats until the end of the dataset. The observations and parameters storage can be a computational problem during the incremental B.A. process. Therefore, a further step of data reduction should be necessary to evaluate which of the previous data are essential to be maintained, need to be updated or become outdated and can be discarded. This step will not be approached in this work.

\section{EXPERIMENTS AND RESULTS}

\subsection{Dataset}

A sequential data acquisition with the PMTS (Section 2) was performed in a narrow path $\left(22^{\circ} 07^{\prime} \mathrm{S}, 51^{\circ} 24^{\prime} \mathrm{W}\right)$ covered with sparse vegetation areas (Eucalyptus trees and ground vegetation) and urban features (small building, light poles and traffic signs). A $10 \mathrm{~m}$ trajectory was traversed, resulting in a set of 28 images (14 omnidirectional images) and 28 groups of initial EOPs from the PMTS navigation system (Ublox NEO$6 \mathrm{M} / \mathrm{MPU6050)}$. The pixel size in object space units of the fisheye images in this dataset range between 1 and $80 \mathrm{~cm}$. The initial navigation data were obtained with a positional accuracy varying between 1 and $5 \mathrm{~m}$. Larger systematic errors in the Ublox NEO-6M positioning are usually obtained in height (Campos et al., 2018). Thus, an initial correction value $(\Delta \mathrm{Z} \approx 5 \mathrm{~m})$ was applied in the heights of all camera positions $\left(\mathrm{Z}_{0}\right)$ estimated with Ublox NEO-6M. The $\Delta \mathrm{Z}$ value (constant) was computed considering the height of GCPs in the begging of the trajectory aligned with the camera center position, which enable a better estimation of the initial 3D ground coordinates. 
The Ublox NEO-6M data were acquired in NMEA format (latitude, longitude) and converted to UTM $(\mathrm{E}, \mathrm{N}, \mathrm{h})$, which is compatible with the coordinate system that is used for GCPs.

A set of 12 GCPs (6 appearing in the image of each sensor) were obtained from an existing terrestrial LASER point cloud (collected with Leica ScanStation P40). A standard deviation of $5 \mathrm{~cm}$ was assigned for the GCPs in the object space which is compatible with the point cloud accuracy and resolution. The 3D ground coordinates were measured in the LASER point cloud using Cloud Compare and Fugro Viewer software. All corresponding GCPs were identified and manually measured in the images. The coordinates in the image and object spaces were previously informed for the incremental process. The 12 GCPs were located in the begging of the trajectory, but along the trajectory the visibility of the reference points decreased until the total absence of GCPs. At the end of the trajectory a bundle adjustment was performed with 4 check points measured in the last six images to estimate the accuracy of the $3 \mathrm{D}$ point cloud generated. The coordinates of the check points used as reference were also measured in the LASER point cloud

A simulation of a real-time photogrammetric process was performed considering the dataset above described and the workflow defined in Section 4. The performance assessment of the incremental bundle adjustment using PMTS data was done mainly focusing on the positional accuracy of the trajectory and the accuracy of the $3 \mathrm{D}$ coordinates map.

\subsection{PMTS trajectory and the 3D ground coordinates accuracy}

The accuracy of the camera stations (trajectory) was estimated by comparing the results from the incremental bundle adjustment with reference values (a simultaneous bundle block adjustment with PhotoScan). Figure 2 presents the trajectory estimated with the Ublox GPS receiver (red), the estimated trajectory with the incremental bundle adjustment for sensor 1 (gray) and sensor 2 (blue) and the ground reference (green). The trajectory was estimated with a positional accuracy (RMSE) of $0.25 \mathrm{~m}, 0.16 \mathrm{~m}$ and $0.15 \mathrm{~m}$ for $\mathrm{E}, \mathrm{N}$ and $\mathrm{h}$ respectively, resulting in a planimetric accuracy of $0.29 \mathrm{~m}$ and planialtimetric accuracy of $0.33 \mathrm{~m}$

The accuracy of the 3D ground coordinates was assessed with 4 checkpoints, specially positioned at the end of the trajectory path (last 6 images). Table 1 presents the statistics, including the mean $(\overline{\mathbf{x}})$ standard deviation $(\sigma)$ and RMSE of the discrepancies between the 3D coordinates of the 4 check points measured in a LASER point cloud (reference) and the coordinates estimated by the incremental bundle adjustment. Therefore, the $3 \mathrm{D}$ ground coordinates $(\mathrm{E}, \mathrm{N}, \mathrm{h})$ of the points detected with $\mathrm{SIFT}_{\mathrm{RFS}}$ (tie points) were estimated at the end of the trajectory with a positional accuracy (RMSE) of $0.8 \mathrm{~m}, 0.7 \mathrm{~m}$ and $0.5 \mathrm{~m}$ for $\mathrm{E}, \mathrm{N}$ and $\mathrm{h}$ respectively, resulting in planimetric accuracy of $1 \mathrm{~m}$.

\begin{tabular}{|c|c|c|c|c|}
\hline Statistics & $\mathbf{E}(\mathbf{m})$ & $\mathbf{N}(\mathbf{m})$ & $\mathbf{h}(\mathbf{m})$ & TOTAL (m) \\
\hline$\overline{\mathbf{x}}$ & -0.245 & 0.190 & 0.304 & - \\
$\sigma$ & 0.876 & 0.752 & 0.356 & - \\
RMSE & 0.798 & 0.679 & 0.433 & 1.02 \\
\hline
\end{tabular}

Table 1. Statistics: mean, standard deviation and RMSE of the checkpoints

The results presented in Figure 2 and Table 1 show the drift of the solution along the trajectory caused by different sources of errors. The 3D map accuracy is affected by the sequential error propagation. First of all, the MPU5060 data was very noisy after 6 meters (after sensor pose n. 7) of PMTS traversing due to the platform movements. The error in the initial EOPs directly affected automatic extraction of image observations ( $\mathrm{SIFT}_{\mathrm{RFS}}$ ) and the initial map estimation. Regarding SIFT $_{\mathrm{RFS}}$ performance, the reprojection errors of image observations are smaller than 2 pixels. However, the errors in the initial EOPs affected the precision of the epipolar line projection, which usually results in a poor geometric distribution of the match points in the keyframes. The outliers and the weak geometry of match points are also mentioned in related works, as the main reasons for the drift of the incremental bundle adjustment solution (D. Scaramuzza et al., 2009). The space intersection model used to compute the initial 3D ground coordinates of the tie points also depends directly on initial EOPs accuracy. Thus, the 3D ground coordinates estimated at the end of the trajectory presented a standard deviation between $3 \mathrm{~m}$ and $5 \mathrm{~m}$. At this point (instant $\mathrm{t}=10$ ), the number of GCPs is reduced and far from the sensor, for instance, 2 GCPs for each sensor side (maximum), which hinders a more accurate solution in the incremental process.

Despite of the GPS/IMU noises and the weak geometry of some images, the proposed methodology was able to weight the observations properly and recover the solution, as presented in Figure 2. For instance, the residuals in the sensor position start

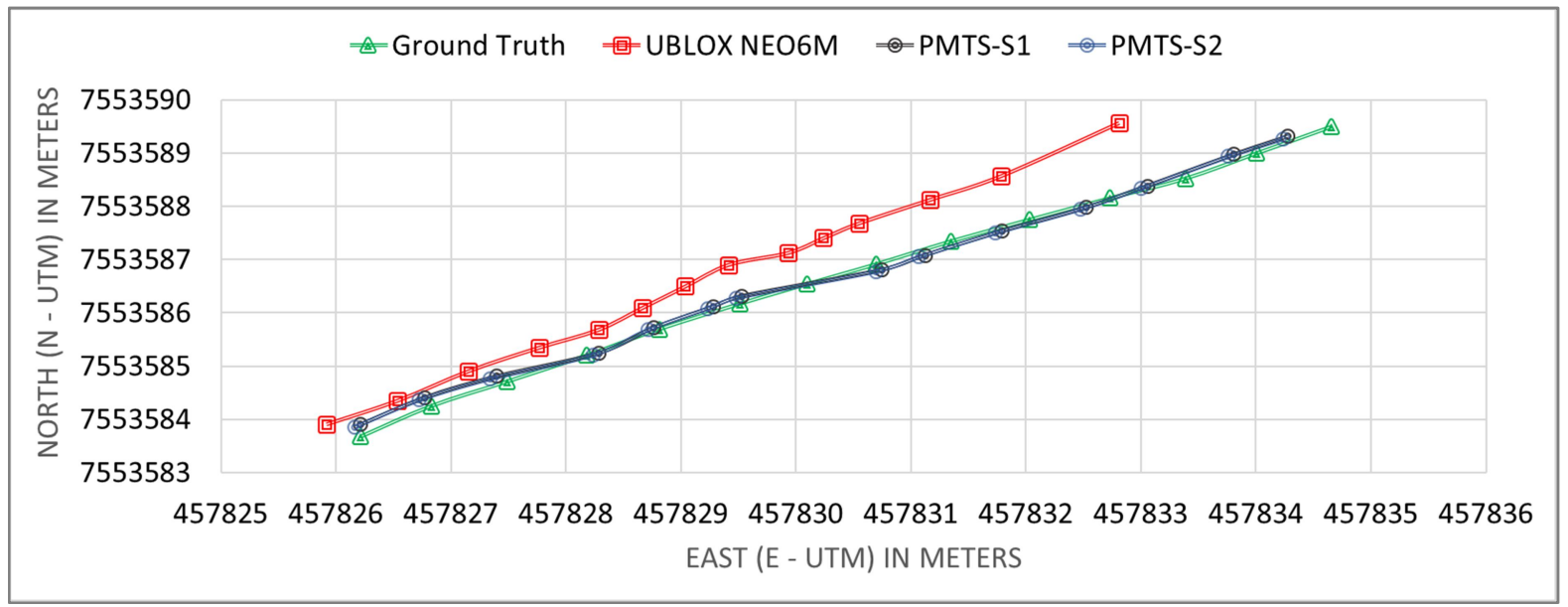

Figure 2. Sensor trajectory estimated by simultaneous bundle adjustment with PhotoScan (taken as ground reference), Ublox GPS receiver and in the incremental bundle adjustment using PMTS data along the trajectory. 
to increase between instant $\mathrm{t}=7$ and $\mathrm{t}=10$ (nearly to $1 \mathrm{~m}$ in $\mathrm{E}$ ), since some keyframes with weak geometry were included. However, the solution for instants $t=11$ to $t=14$ are close to the reference values with a planimetric error of $0.5 \mathrm{~m}$, approximately, which is consistent with the average trajectory accuracy (RMSE) estimated.

Furthermore, the proposed incremental BA methodology was evaluated considering a data acquisition performed with the proposed PMTS in an outdoor area (sidewalk) with areas covered with vegetation, which can be considered more challenging due to obstruction of the GNSS signals. The PMTS trajectory was estimated with the incremental bundle adjustment with an accuracy (RMSE) of $0.33 \mathrm{~m}$. These results showed that passive optical systems combined to low-cost navigation sensors can be considered a feasible alternative to estimate sensor position in covered areas, for instance, natural or planted forests, where direct methods based only on GNSS and IMU are not often available or accurate due to GPS signal obstruction.

\section{CONCLUSIONS}

This work presented a feasibility study of an incremental bundle adjustment methodology for real-time orientation and mapping, based on ISO approach and using data coming from a low-cost and lightweight PMTS acquired in an outdoor test area. The experimental assessment showed the potential of low-cost systems based on MEMS measurement, as source of initial information for the photogrammetric processing. For instance, methodologies based on the use of relative orientation parameters estimated from GNSS/IMU-MEMS, such as $\mathrm{SIFT}_{\mathrm{RFS}}$, can provide more accurate and optimized solutions. However, the MEMS navigation system used still provides high level of errors and noise. These errors can result in a quick drift of the solution, requiring future assessments focusing in a stochastic treatment of the initial information provided.

This optical-based omnidirectional system offered an option for mobile mapping over covered areas, enabling more flexible mobile mapping systems for CRP applications. Since Ricoh Theta $\mathrm{S}$ cameras are arranged in the same structure, the relative orientation stability constraints were introduced to improve the consistency between the EOPs estimative for an omnidirectional image. For instance, the estimated positions of each Ricoh Theta $\mathrm{S}$ camera station vary only $2 \mathrm{~cm}$ (linear offset). However, at the end of the trajectory, the two sets of EOPs from an omnidirectional image were estimated with discrepancies higher than the expected linear and angular offsets, due to the errors propagation. On average, the EOP estimated for each Ricoh Theta $\mathrm{S}$ camera had a linear offset of $10 \mathrm{~cm}$. Therefore, further investigations are required, aiming a more stable mathematical model.

Many improvements are still required to automate the PMTS process for robust real-time applications. The weak geometry of some points in fisheye images is one of the main reasons for the non-convergence and the instability of the incremental $\mathrm{BA}$ solution. Therefore, the main challenges observed in this study for real-time CRP application relies on the automatic detection of image observations (image matching assessment and geometry) to support a consistence network of matches and the auto-diagnosis to remove or maintain observations (outliers and points with weak geometry).

\section{ACKNOWLEDGEMENTS}

This study was funded by Coordenação de Aperfeiçoamento de Pessoal de Nivel Superior-Brasil (CAPES) (Grants: $88881.135114 / 2016-01 ; \quad 1481339, \quad 1774590 \quad$ and 88887.310313/2018-00, 88881.310314/2018-01), Conselho Nacional de Desenvolvimento Científico e Tecnológico (Grant: 155739/2018-2) Fundação de Amparo à Pesquisa do Estado de São Paulo (FAPESP) under Grant 2013/50426-4 and the Academy of Finland (Grant: 273806).

\section{REFERENCES}

Cheein, A., Steiner, F., Perez Paina, G., Carelli, R., 2011. Optimized EIF-SLAM algorithm for precision agriculture mapping based on stems detection. Comput. Electron. Agric. 78, 195-207.

Campos, M.B., Tommaselli, A.M.G., Castanheiro, L.F., Oliveira, R.A., Honkavaara, E., 2019. A Fisheye Image Matching Method Boosted by Recursive Search Space for Close Range Photogrammetry. Remote Sens. 11.

Campos, M.B., Tommaselli, A.M.G., Honkavaara, E., Prol, F. dos S., Kaartinen, H., El Issaoui, A., Hakala, T., 2018. A Backpack-Mounted Omnidirectional Camera with Off-the-Shelf Navigation Sensors for Mobile Terrestrial Mapping: Development and Forest Application. Sensors 18.

Scaramuzza, D., Fraundorfer, F., Siegwart, R., 2009. Real-time monocular visual odometry for on-road vehicles with 1-point RANSAC, in: 2009 IEEE International Conference on Robotics and Automation. Presented at the 2009 IEEE International Conference on Robotics and Automation, pp. 4293-4299.

Gruen, A., 1988. Towards real-time photogrammetry. Photogrammetria 42, 209-244.

Hughes, C., Denny, P., Jones, E., Glavin, M., 2010. Accuracy of fish-eye lens models. Appl Opt 49, 3338-3347.

Suhr, J. K., Jang, J., Min, D., Jung, H. G., 2017. Sensor FusionBased Low-Cost Vehicle Localization System for Complex Urban Environments. IEEE Trans. Intell. Transp. Syst. 18, 1078-1086.

Lowe, D., 2004. Distinctive Image Features from ScaleInvariant Keypoints. Int. J. Comput. Vis. 60, 91.

Lourenco, M., Barreto, J. P. , Vasconcelos, F. , 2012. sRDSIFT: Keypoint Detection and Matching in Images With Radial Distortion. IEEE Trans. Robot. 28, 752-760.

Marcato Junior, J., Moraes, M.V.A. de, Tommaselli, A.M.G., 2015. Experimental assessment of techniques for fisheye camera calibration. Bol. Ciênc. Geodésicas 21, 637-651.

Mikhail, E.M., Ackerman, f, 1976. Observations and least squares. University Press of America Inc, New York.

Mur-Artal, R., Montiel, J. M. M., Tardós, J. D. , 2015. ORBSLAM: A Versatile and Accurate Monocular SLAM System. IEEE Trans. Robot. 31, 1147-1163.

Ericson, S., Åstrand, B. , 2010. Row-detection on an agricultural field using omnidirectional camera., in: 2010 IEEE/RSJ International Conference on Intelligent Robots and Systems. Presented at the 2010 IEEE/RSJ International Conference on Intelligent Robots and Systems, pp. 4982-4987.

Tommaselli, A.M., Galo, M., De Moraes, M.V., Marcato, J., Caldeira, C.R., Lopes, R.F., 2013. Generating virtual images from oblique frames. Remote Sens. 5, 1875-1893. 